\title{
A Probabilistic Capacity Planning Methodology for Plug-in Electric Vehicle Charging Lots with On-site Energy Storage Systems
}

\author{
I. Safak Bayram, Stuart Galloway, Graeme Burt.
}

\begin{abstract}
Plug-in electric vehicles (PEV) have gained popularity to support environmental sustainability and reach net-zero emission goals. However, accommodating large numbers of PEVs is a complex problem as concurrent PEV demand significantly increases peak demand and stresses supporting network elements. In this paper, we present a large-scale PEV charging lot equipped with an on-site storage. Power drawn from the grid is utilized to meet customer demand and charge the storage unit which, in return, is employed to lower peak load and demand charges. By considering the probabilistic nature of the customer demand, the proposed architecture is modelled by a Markov-modulated Poisson Process and a matrix-geometric based algorithm is developed to solve the associated capacity planning problem. Station outage probability (defined as the probability of not serving PEV demand) is used as the main metric to size station resources. Case studies show that by accounting for the statistical variations in customer demand, the power required for the station is significantly less than the sum of chargers' rated power. In addition, on-site storage can considerably reduce the stress on the supporting grid components and lower stations' running cost.
\end{abstract}

\section{Index Terms}

Energy storage systems, Plug-in electric vehicles, markov-modulated poisson process, demandcharge management.

I. Safak Bayram, Stuart Galloway, and G. Burt are with the Department of Electronic and Electrical Engineering, University of Strathclyde, Glasgow, G1 1XQ, UK, e-mail: \{safak.bayram,stuart.galloway,graeme.burt\}@strath.ac.uk. Corresponding Author: I. Safak Bayram 


\section{INTRODUCTION}

To meet climate change targets and improve urban air-quality, there has been a motivation to push plug-in electric vehicles (PEV) into mainstream adoption. This trend is further supported by public policies around the world to promote PEV ownership [1]. The UK government has announced a ban on the sale of new internal combustion engine vehicles (ICEV) by 2040 [2] and similar targets have been set by several countries including France, Norway, and India to be implemented via either substantial taxation or regulatory measures. On the other hand, the success of PEV adoption relies on the capability of the power grid to provide enough coverage and capacity to fuel PEVs on the road. Due to the increasing shares of intermittent variable generation, power grids are becoming more stressed, hence, the transition towards electric mobility requires a careful investigation of potential disruptive impacts of PEV chargings [3]. Previous research shows that even small clusters of simultaneous PEV charging could lead to grid problems such as premature aging of distribution transformers [4], power quality deterioration [5], shortages [6], and possibly increase peak system demand. As the transportation and power sectors become tightly coupled, laying the foundations for sustainable charging infrastructures is a critical step in reducing the carbon footprint of the transportation sector.

The UK government has rolled out aggressive plans to expand charging networks to support millions of PEVs as a part of broader efforts to reduce all greenhouse gas emissions to net zero by 2050 (see [7]). In parallel, the Scottish government has deployed one thousand charging nodes and the average distance from any given location to the nearest charger is less than three miles [8]. In the United States, the West Coast Green Highway project hosts chargers at every 25 miles and covers nearly 800 miles of this highway network [9]. Although current charging networks seem to provide reasonable coverage, charging capacity should be upgraded commensurate with PEV penetration rates. According to International Energy Agency, pure electric vehicle sales is expected to reach 150 million globally by 2030 [10]. Primary drivers for this disruptive change includes sharp reductions in the cost of lithium-ion battery technology and increasing number of vehicle preferences comparable to ICEV counterparts [11].

At the moment, there are three options for PEV charging [13], [14]. First is the slow charging option that often uses level 1 chargers with rated power up to $1.92 \mathrm{~kW}$. Considering the average battery size of current PEV models $(20+\mathrm{kWh})$, this option is suitable for domestic/garage use cases. Second option, on the other hand, uses level 2 chargers (up to $19.2 \mathrm{~kW}$, but mostly around 
TABLE I: Electric Vehicle Charger Type and Costs [12]

\begin{tabular}{llll}
\hline Charger Type & Unit Cost Range & Unit Installation Costs & Location \\
\hline Level 1 & $\$ 300-\$ 1500$ & $0-\$ 3000$ & Primarily home \\
Level 2 & $\$ 400-\$ 6500$ & $\$ 600-\$ 12700$ & Home, workplace, public \\
DC Fast & $\$ 10000-\$ 40000$ & $\$ 4000-\$ 51000$ & Public and intercity \\
\hline
\end{tabular}

6-7 $\mathrm{kW}$ ) and they are mostly deployed at parking lots (e.g., workplaces, shopping malls, etc.) as PEVs typically remain stationary for several hours. Note that these two methods use AC supply and transfer power via PEV's onboard charger. Third charging option is DC fast charging which mimics a gas station. Notice that typical DC chargers are rated at $50 \mathrm{~kW}$, while recent ultrafast models can transfer power at $350 \mathrm{~kW}$. Nevertheless, with a typical $50 \mathrm{~kW}$ charger it would take around half an hour to deliver enough charge for a 100-mile trip. In all cases, simultaneous PEV demand adds a sizeable load on the grid and sustainable charging infrastructures and smarter charging strategies are needed to (1) keep power grids within operational limits, (2) minimise required system upgrades, and (3) lower service cost for end-users. The capital cost for charging equipments is presented in Table I. It can be seen that the economies of electric vehicle charger types vary widely due to different architectures, charging rates, and the cost related to installation sites.

In this paper, a large-scale PEV charging lot equipped with Level 2 chargers and an energy storage unit is presented. In the model charging station draws power from the grid to charge PEVs and the storage unit whenever possible. Stored energy, in return, is discharged during peak hours to minimise peak demand and lower demand charges. By modeling arrival, departure, and demand profiles by Poisson processes, the overall system is modeled with a Markov-modulated Poisson Process (MMPP). Energy storage sizing problem is computed by solving steady-state system probabilities since system states are represented by number of active PEVs in the system and onsite storage charge level. A matrix-geometric based algorithm to solve steady-state distributions and calculate energy storage size. Through case studies with actual electricity prices, the role of of storage unit in lowering peak load and demand charges is explored in detail.

The literature on charging station design and operation can be grouped into two categories. The first group includes studies from technology development standpoint and more recent attention has focused on improving the energy conversion efficiency and fault-tolerant ability of power 
conversion systems for PEV chargers. It is noteworthy that the lifetime of PEV batteries is closely linked to the charging and discharging profile of batteries [15]. Therefore, battery chargers play a critical role in lifecycle assessment and economic viability of PEVs. As discussed earlier, on-board chargers are located inside the vehicle and enables AC power transfer, however with limited energy transfer capability [16]. To increase charging rates without adding extra weight on the PEV, integrated battery charger and drive system that reutilises electric traction motor are developed [17]. Design and operation of renewable energy based PEV charging stations has gained popularity [18], [19]. In [18], a two-stage optimisation framework for PEV charging station is presented. In the first stage, day-ahead hourly renewable energy generation and customer demand are forecasted. In the second stage, charging rate of each PEV is calculated according to actual PEV load and solar generation. The goal was to minimise reliance on the power grid and serve as much as possible from the renewable generation. In [19], a scheduling model is presented to schedule PEV loads at a solar charging infrastructure. The proposed framework aims to shift PEV load to increase utilisation of solar energy generation. Furthermore, detailed overview of PEV charging infrastructures are presented in the following review articles [20], [21], and [22].

Ultra-fast charging architectures have been the focus of many studies [14], [23] and a number of charger models were announced by major manufacturers such as Tesla and ABB [24]. Stations containing multiple fast or ultra-chargers are typically connected to medium-voltage utility supply via a 3-phase step-down transformer that typically provides power at $480 \mathrm{~V}$ (line to line). Such stations may be in the form of $A C$ or DC coupling. AC coupled systems include mature converter, switchgears, and protection devices, hence well-established standards exist for ac distribution stations. However, multiple conversion stages reduce the system efficiency of $A C$ systems. Also, controlling $A C$ stations are more complieated than DC ones due to the requirement of reactive power control, inverter synchronisation, and other complex control needs. DC distribution model, however, requires a service transformer and a low voltage rectifier. The main advantage of DC system is higher system efficiency due to elimination of $A C / D C$ and DC/AC conversions.

The work presented in this paper falls into the second group which focuses on economic operation of charging stations with an on-site storage and large numbers of Level 2 chargers [25]-[27]. In this group, a critical component of the charging facility is an on-site energy storage unit which is typically used for peak shaving, reduce demand charges, and provide additional 
income via energy market participation. In [25], an optimal sizing approach for energy storage systems in fast charging stations is proposed. Customer demand is determined based on driving patterns and an optimal storage size is determined based on cost minimisation. In [27], authors present an optimisation framework for optimal bidding strategy in a day-ahead electricity markets for a PEV charging station with an on-site storage. In [26], a resource allocation framework for a network of fast-charging stations with storage units is presented to lower peak PEV demand. By making Poisson assumptions on PEV arrival and departures, charging station is modelled with a Markov chain and system performance is calculated with numerical methods. Queuing systems and stochastic modelling have been used in several other studies to capture the probabilistic nature of the customer demand such as different battery packs, technologies, and customer arrival and departure processes. In [28], a queuing model is used to calculate waiting times and service quality for a number of charging stations located in Ohio by using actual traffic traces. In [29], a charging station is modeled using a queuing model and captured the effect of constant current constant voltage charging on customer waiting times in the station. One important system parameter is customer arrival and charging demand statistics. In [30], using actual PEV charging data (level 2 chargers) from a major North American University Campus between 2010-2015, a Markovian model for representing the charging behaviour of PEV owners is presented. The results show that PEV owners not necessarily fully charge their batteries, hence, service duration are shorter than expected. On the other hand, most of the studies assume Poisson arrival and departure processes (number of PEVs arriving in an hour) in the models due to relatively simplified mathematical analyses [31].

Currently, high demand charges constitute a large portion of charging station's monthly electricity bills and narrows station's profit margins [32]. Demand charges are tariffs that are typically used for commercial and industrial customers to limit their peak consumption by introducing an extra fee commensurate to the highest level of consumption typically during any 15 minutes within each month. In an actual operational charging station with two $50 \mathrm{~kW}$ chargers located in San Diego, California, the following electricity bill was issued in June 2016 [33]. The energy cost was 284 USD, while demand charges due to $87 \mathrm{~kW}$ peak power reached to 2910 USD, making up $91 \%$ of the electricity bill. Demand charges at this level not only threaten business models but also may negatively affect PEV penetration if prices are reflected to customers.

In this paper, a stochastic model for charging lot with an on-site storage is developed. Based on Poisson arrival, departure and demand statistics, an MMPP model is developed and storage 
sizing is solved by computing the model's steady-state probability distributions. A number of case studies are presented to highlight the interplay between storage size, customer load, and power drawn from the grid. Results show that on-site storage units can significantly lower station's operational costs and peak station load.

\section{SySTEM DESCRIPTION}

We consider a group of $N$ chargers located in a parking lot to serve the demand of plug-in electric vehicles. The charging service is provided by (i) power drawn from the grid and (ii) an energy storage system (ESS) shared by all customers. The total system charging power at time $t$ is denoted by $\nu_{t}$ and ESS charge level is $i(t)$, for $t \in \mathbb{R}^{+}$. Grid power is used to serve vehicles and charge the storage unit whenever possible. When the aggregate demand is higher than $\nu_{t}$, then ESS is discharged to serve customers unless $i(t)=0$. We assume that PEVs arrive at the parking lot according to a Poisson process with rate $\lambda$. The average parking duration is assumed to follow an exponential distribution with rate $\mu$, and when a PEV is parked, its power demand is modelled by a Poisson process with rate $\beta$ (for similar Poisson assumptions see [34], [26], and [31]). The rationale behind the last assumption is that the charging session does not necessarily take the same amount of time as the charging duration. In addition, a parking coordinator may be applying smart charging to adjust charging power according to network conditions, battery state of charge, ambient temperature, or electricity prices (see [35]-[38]). Moreover, if an arriving customer finds all system resources in-use, then an outage event occurs. In the model, outage probability naturally serves as the main performance metric. Figure 1 depicts the system being modeled.

Note that PEV arrivals act independently, and the proposed system's state space $\{0,1, \ldots, N\}$ is a birth-death process. The composite model for $N$ slot charging station is shown in Figure 2. Since at each system state, aggregate demand has a state-dependent Poisson rates (e.g., $\beta$, $2 \beta$, etc.), assumptions discussed above further give rise to an MMPP model which will be used to model charging station operation dynamics and to calculate energy storage size. More detailed discussion is given in the next section. For the given system model, note a natural assumption that the system operates in steady-state and in a stable region. For this, average aggregate PEV load should be strictly less than the station capacity. Hence,

$$
N \beta\left(\frac{\lambda}{\lambda+\mu}\right)<C_{t}
$$




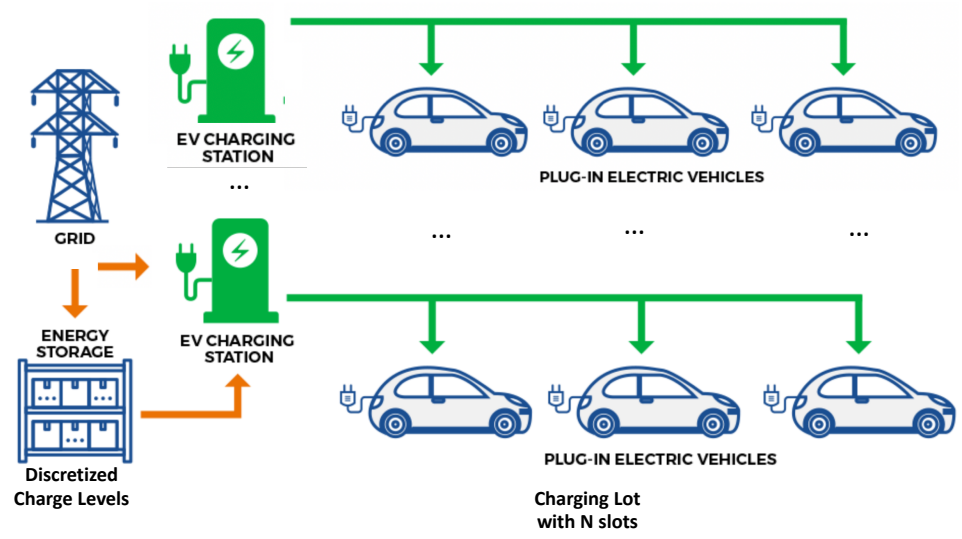

Fig. 1: Plug-in electric vehicle charging lot with $N$ chargers.



Fig. 2: Composite model for charging lot with $N$ chargers.

or utilization parameter $\rho$ can be written as,

$$
\rho \equiv N \beta\left(\frac{\lambda}{\lambda+\mu}\right) \frac{1}{C_{t}}<1
$$

Moreover, the following assumptions are made for the energy storage unit: (i) Energy rating (in $\mathrm{kWh}$ ) or the size of the energy storage is denoted by $B$; (ii) Energy storage charge-discharge efficiency is denoted by $\eta$ which takes values between 0 and 1 . This parameter reflects the percentage of transferred or stored energy after losses are excluded; (iii) Due to leakage, storage units loses a small percentage of energy due to leakage. To simplify notations, such losses due to dissipation are ignored. During any time of the station operations, three cases define the storage unit's state of charge level: (i) ESS is fully discharged $(i(t)=0)$ and total demand is more than $\nu_{t}$; (ii) ESS is fully charged $\left(i(t)=1\right.$ ), and the total demand is less than $\nu_{t}$; and (iii) ESS is in a partially charged state $((0<i(t)<1))$ with any system demand. In the first two cases, the rate of change in storage charge level would be zero. However, for the third case, the rate of change in energy storage charge level would be the difference between charging power $\nu_{t}$ and the total 
demand on the system, e.g., $\frac{d i(t)}{d t}=\eta\left(\nu_{t}-\sum_{n} L_{n}(t)\right)$, where $\left.L_{n}(t)\right)$ denotes total demand when at time $t$ and $i(t)$ is the ESS charge level.

Due to the probabilistic nature of the system, by choosing any storage capacity $B$, only stochastic guarantees can be provided for the system reliability and there is always a chance of system outage, which happens when an idle system resources fall below the total demand. By noting that $i(t)$ shows the ESS charge level that is needed to an avoid outage, a system outage occurs when the system load exceeds the maximum available ESS size $B$. Hence, let us define $\varepsilon$-outage storage capacity, denoted by $B(\varepsilon)$, as the minimum $B$ choice corresponding to which the probability of outage is upper limited by $\varepsilon \in(0,1)$, i.e.,

$$
B(\varepsilon)=\left\{\begin{array}{ll}
\min & B \\
\text { s.t. } & \mathbb{P}(i(t) \geq B) \leq \varepsilon
\end{array} .\right.
$$

The primary goal of this paper is to calculate the $\varepsilon$-outage storage capacity $B(\varepsilon)$ based on grid capacity $\nu_{t}$, number of users, and other system parameters. For simplicity in mathematical expressions, the ESS parameter is scaled and instead of $B / \eta B$ is redefined as the maximum amount of energy that can be stored. Moreover, in power system operation and planning, statistical guarantees are typically calculated based on "busy hour" period [39]. Hence, in the rest of the paper, we drop time index $t$ and assume the calculations are for peak hour statistics. Moreover, to be consistent with other system parameters, we replace grid capacity $C$ with parameter $\nu$.

\section{A. Markov-modulated Poisson Process Model}

Recall that the main objective is to analyse the statistical behaviour of the energy storage charge level. Let $n \in 0, \ldots, N$ denote the number of PEVs that are actively getting charged. We further let $p_{i n}$ denote the joint probability that ESS charge level is $i$ with $n$ are active:

$$
p_{i n}=\mathbb{P}(\text { ESS charge level }=i, n \text { active customers }) .
$$

To that end, the probability that ESS charge level is $i$ can be written as

$$
p_{i}=\sum_{n=0}^{N} p_{i n}
$$

Aforementioned assumptions allow us to model the system with a two-dimensional continuoustime Markov chain as shown in Figure 3. System states are represented by a doublet $(i, n)$, where 


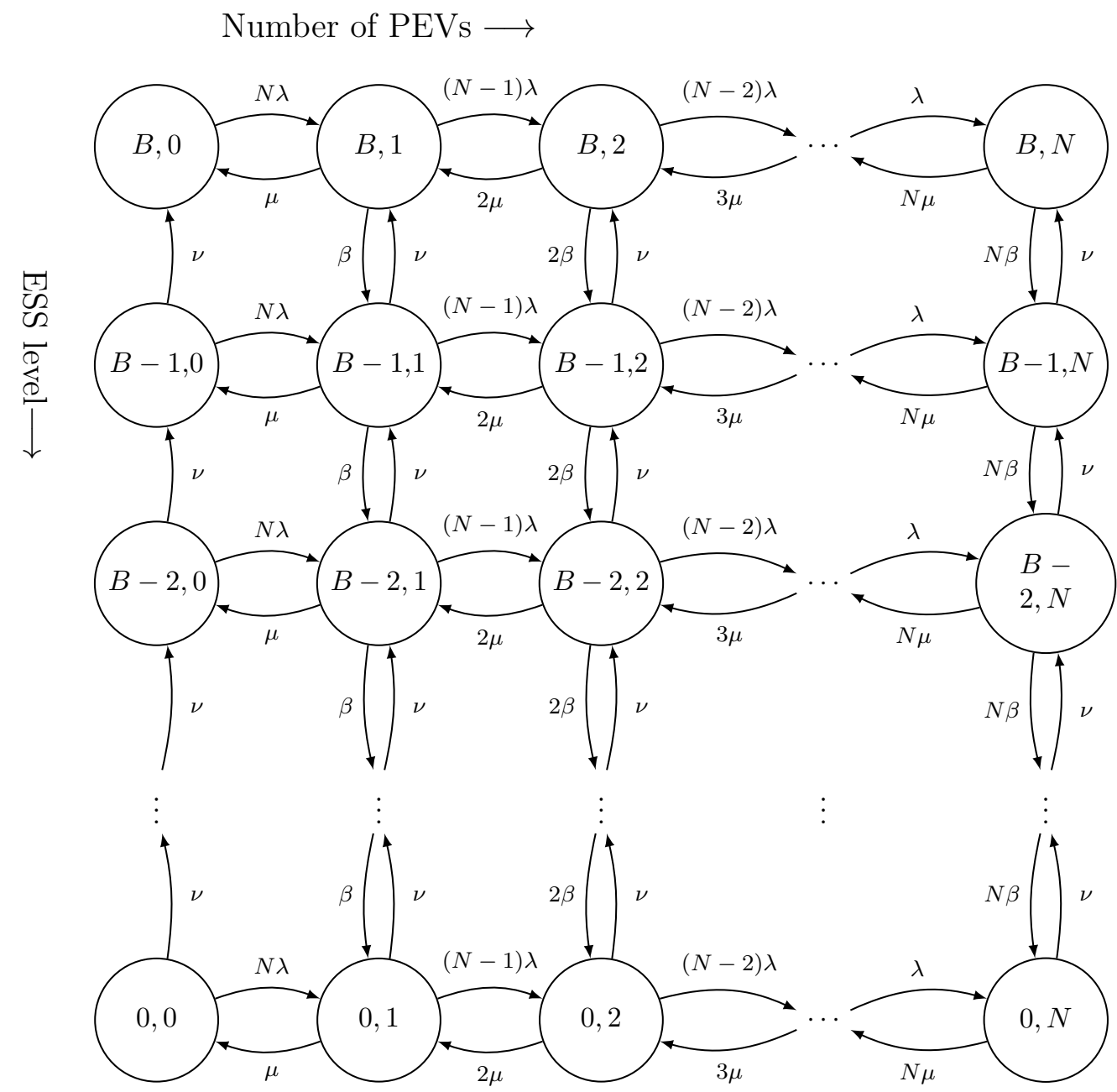

Fig. 3: State space representation of the station model. Vertical state transitions represent energy storage charge-discharge, while horizontal ones depicts vehicle arrival and departure.

the first dimension varies from 0 to $B$ (vertical transitions) and the second dimension takes values from 0 to $N$ (horizontal transitions). Note that vertical transition represent ESS charge and discharge, while horizontal transitions show PEV arrival and departure. Moreover, transitions rates (e.g., $\lambda, \mu$, etc.) are based on the Poisson assumptions made earlier. It is noteworthy that, in our calculations, we first will assume that the energy storage has an infinite size. Whereas the energy storage size will be calculated based on the overflow probability as given in (3). 


\section{B. Matrix Geometric Approach}

To solve steady-state probabilities given in (5), an algorithmic technique called matrix geometric approach has been employed [40], [41]. As a first step, we set up balance equations for the two-dimensional state space to form the system of equations in a desired matrix form. For instance, for the last row $(i=0)$ of MMPP model (see Figure 3), there are three state transition intervals and the balance equation is

$$
N \lambda p_{00}=\mu p_{01}+\nu p_{10}
$$

To apply matrix geometric approach, (6) is rewritten as

$$
p_{00}=(1-N \lambda) p_{00}+\mu p_{01}+\nu p_{10}
$$

For the second interval $i=0,1 \leq n \leq N-1$, the general balance equations are given by

$$
((N-n) \lambda+n \mu+n \beta) p_{0 n}=\left((N-(n-1)) \lambda p_{0 n-1}+(n+1) \mu p_{0 n+1}+\nu p_{1 n}\right.
$$

Similar to the case above, (8) is rewritten as

$$
p_{0 n}=(N-(n-1)) \lambda p_{0 n-1}+(1-(N-n) \lambda-n \mu-n \beta) p_{0 n}+(n+1) \mu p_{0 n+1}+\nu p_{1 j}
$$

The third case includes the rightmost boundary states where $i=0$ and $n=N$. The balance equation in this case is

$$
(N \mu+N \beta) p_{0 N}=\lambda p_{0 N-1}+\nu p_{1 N}
$$

This equation can be transformed into the following form to be used in matrix geometric calculations

$$
p_{0 N}=\lambda p_{0 N-1}+(1-N \mu-N \beta) p_{0 N}+\nu p_{1 N} .
$$

Balance equations for the remaining parts of the Markov chain can be written in a similar manner to (7), (9), and (11), however, by further including vertical state probability transitions. To proceed with matrix-geometric approach, we define a set of $(N+1)$-element row vectors $\boldsymbol{p}_{i}$ as

$$
\boldsymbol{p}_{i} \equiv\left[p_{i 0}, p_{i 1}, \ldots, p_{i N}\right]
$$

Moreover, equations (7), (9), and (11) can be further written in a compact matrix-vector equation. For $\boldsymbol{p}_{0}$, we have

$$
\boldsymbol{p}_{0}=\boldsymbol{p}_{0} B_{0}+\boldsymbol{p}_{1} B_{1}
$$


where $(N+1) \times(N+1)$ matrices $B_{0}$ and $B_{1}$ are readily given by

$$
B_{0}=\left[\begin{array}{ccccc}
(1-N \lambda) & N \lambda & 0 & \cdots & 0 \\
\mu & (1-\mu-(N-1) \lambda-\beta) & (N-1) \lambda & \cdots & 0 \\
0 & 2 \mu & (1-2 \mu-(N-2) \lambda-2 \beta) & \cdots & 0 \\
\vdots & \vdots & \vdots & \ddots & \vdots \\
0 & 0 & 0 & \cdots & (1-N \mu-N \beta)
\end{array}\right]
$$

and $B_{1}$ is a diagonal matrix the elements of which are composed of vertical transition rate $\nu$ shown in Figure 2. Matrices containing the transition rates for the rest of rows of the Markov chain given in Figure 3 can be written similar to (14). However, note that transitions for intermediate rows, i.e., $\boldsymbol{p}_{i>0}$, further includes vertical transitions to previous row. Hence, a complete set of balance equations for the remaining rows can be constructed from the matrix recurrences relation with three matrices such as $A_{0}, A_{1}$, and $A_{2}$, that is,

$$
\boldsymbol{p}_{i}=\boldsymbol{p}_{i-1} A_{0}+\boldsymbol{p}_{i} A_{1}+\boldsymbol{p}_{i+1} A_{2}, \quad \text { for } i>0 .
$$

Recall the assumption that the energy storage capacity is initially assumed to be infinite. Hence, (12) is updated and an infinite-dimension $\boldsymbol{p}$ is introduced as

$$
\boldsymbol{p}=\left[\boldsymbol{p}_{0}, \boldsymbol{p}_{1}, \boldsymbol{p}_{2}, \ldots, \boldsymbol{p}_{i}, \ldots\right]
$$

Then, similar to (13), it is easy to see that,

$$
\boldsymbol{p}=\boldsymbol{p} P,
$$

where matrix $P$ is a stochastic matrix with infinite-dimension and called the transition probability matrix, with each row summing to one. To that end, it can be noticed that the matrix $P$ is concatenated from previously constructed $(N+1) \times(N+1)$ submatrices, namely, $B_{0}, B_{1}, A_{0}$, $A_{1}$, and $A_{2}$, in the following repetitive form

$$
P=\left[\begin{array}{ccccc}
B_{0} & A_{0} & 0 & 0 & \cdots \\
B_{1} & A_{1} & A_{0} & 0 & \cdots \\
0 & A_{2} & A_{1} & A_{0} & \ldots \\
0 & 0 & A_{2} & A_{1} & \cdots \\
0 & 0 & 0 & A_{2} & \cdots \\
\cdots & \ldots & \ldots & \ldots & \ddots
\end{array}\right]
$$

At this point, it is worth mentioning that computation of the energy storage size as shown in (3) becomes finding minimum $i$ which satisfies $\epsilon=1-\sum_{i} \boldsymbol{p}_{i}$. Next, we present a methodology to solve probability distribution function for the energy storage system. 


\section{Algorithmic Solution Technique}

We follow the algorithmic probability solution method developed by Neuts in [40] showed that the solution to $p_{i}$ in (16) is written as

$$
\boldsymbol{p}_{i+1}=\boldsymbol{p}_{i} R \quad i \geq 0,
$$

where $R$ is a $(N+1) \times(N+1)$ matrix. In general, this matrix has a non-negative solution to the following matrix equation

$$
R=\sum_{k=0}^{\infty} R^{k} A_{k} .
$$

Then, calculation of $\boldsymbol{p}_{i}$ in (16) is equivalent to finding the minimal nonnegative solution to $R$ matrix ${ }^{1}$. A recursive calculation method is used to find the matrix $R$. As a first step, (20) is rewritten as

$$
R\left[I-A_{1}\right]=\sum_{\substack{k=0 \\ k \neq 1}}^{\infty} R^{k} A_{k},
$$

where $I$ is an identity matrix. Next, by multiplying both sides of equation (21), we get

$$
R=\sum_{\substack{k=0 \\ k \neq 1}}^{\infty} R^{k} A_{k}\left[I-A_{1}\right]^{-1} .
$$

For a trial initial solution $R=0, R$ can be solved iteratively giving,

$$
R=\left[A_{0}+R^{2} A_{2}\right]\left[I-A_{1}\right]^{-1} .
$$

Now that $R$ is found, we proceed with calculation of $\boldsymbol{p}_{0}$. In general form, $\boldsymbol{p}_{0}$ can be obtained by solving

$$
\boldsymbol{p}_{0}=\boldsymbol{p}_{0} B(R),
$$

where,

$$
B(R)=\sum_{k=0}^{\infty} R^{k} B_{k} .
$$

For the system modelled in this paper, $B(R)$ is composed of two components, i.e., $B(R)=$ $B_{0}+R B_{1}$. Recall that the sum of probabilities for each $\boldsymbol{p}_{0}$ should sum up to one, i.e., $\sum p_{i}=1$. Hence, we need to perform normalisation to satisfy basic probability condition. A straightforward way is to sum the probabilities. Let $T$ denote the sum of $\boldsymbol{p}_{i}$ for $\forall i$. Then the computational results

\footnotetext{
${ }^{1}$ It is worth discussing that, according to Ref. [42], the eigenvalues of matrix $R$ must lie within a unit disk. Eigenvalues with spectral radius more than 1 implies instability in the queuing mechanism and violates stability condition (2).
} 




Fig. 4: Illustrative Example: $\mathrm{M} / \mathrm{M} / 1$ system state.

can be normalised by dividing each probability by $T$. To that end, matrix geometric approach to calculate energy storage size can be summarised as below:

1) Calculate matrix $R$ by solving the equation given in (20) using iteration (22).

2) Calculate $\boldsymbol{p}_{0}$ by solving the eigenvector equations given in (24).

3) Calculate $\boldsymbol{p}_{i}$ by solving (19).

4) Normalize $\boldsymbol{p}_{0}$ and $\boldsymbol{p}_{i}$ by dividing each probability by sum of all probabilities.

5) Calculate minimum energy storage size that satisfies $\epsilon=1-\sum_{k=0}^{i} \boldsymbol{p}_{i}$.

In the next section, we provide case studies to better illustrate proposed model.

\section{NUMERICAL EXAMPLES}

\section{A. Toy Example}

As a toy example to apply matrix geometric method, we consider an M/M/1 queueing system [43] which has a state space represented by an integer $L \geq 0$. Customer arrivals are modeled by a Poisson process with rate $\lambda$ and, similarly, departures follow a Poisson process with rate $\mu$. State diagram for this toy example is depicted in Figure 4. In this case, since transitions are limited to neighboring states, matrices $A$ and $B$ become scalars, that is, $A_{0}=\lambda, A_{1}=1-\lambda-\mu$, $A_{2}=\mu, B_{0}=(1-\lambda)$, and $B_{1}=\mu$. Moreover, the matrix $P$ given in (26) is given by

$$
P=\left[\begin{array}{ccccc}
1-\lambda & \lambda & 0 & 0 & \cdots \\
\mu & 1-\lambda-\mu & \lambda & 0 & \cdots \\
0 & \mu & 1-\lambda-\mu & \lambda & \cdots \\
0 & 0 & \mu & 1-\lambda-\mu & \cdots \\
\vdots & \vdots & \vdots & \vdots & \ddots
\end{array}\right] .
$$

Iteration in (22) can be solved and $R$ becomes

$$
R=\lambda+(1-\lambda-\mu) R+\mu R^{2}
$$


Equation (27) can be solved for $R$ and two roots are obtained: $R=\frac{\lambda}{\mu}$ and $R=1$. Note that second root is not acceptable as it does not lie within the unit disc. To that end, from equation (19)

$$
p_{i+1}=\frac{\lambda}{\mu} p_{i} \quad \text { and } \quad p_{i+1}=\left(\frac{\lambda}{\mu}\right)^{i} p_{0},
$$

which is the standard solution of $\mathrm{M} / \mathrm{M} / 1$ queue [43].

\section{B. Case Studies}

In this section, we present a number of numerical case studies to explain how the proposed system can be used in energy storage sizing and PEV charging lot planning. The parameters are set as follows. It is assumed that charging lot is composed of typical $6 \mathrm{~kW}$ level 2 chargers. Average parking rate is set as one hour $(\mu=1), \beta$ is set as 0.9 , and PEV arrivals per charger vary from $\lambda=0.25$ to $\lambda=0.75$. Moreover, numerical studies are carried our for a range of station sizes starting from $N=50$ to $N=300$. Recall from equation (1) that for stability, power drawn from the grid is rewritten as

$$
C=N \beta\left(\frac{\lambda}{\lambda+\mu}\right)+\Delta
$$

and $\Delta$ is set to a small constant 0.02 . As a first evaluation, we calculate energy storage size for varying station sizes and traffic regime (depicted in Figure 5). As an example, suppose that the station has $N=200$ chargers and peak demand is $\lambda=0.50$. Then to provide an outage probability of $1 \%(\epsilon=0.01)$, energy storage size should be chosen as $136 \mathrm{kWh}$ (depicted in Figure 5(b)). Moreover, two observations can be made from these results. First, as the arrival rate increases, more energy storage capacity is required to provide the same level of outage performance. Second, as the number of chargers increases, the requirement for energy storage size per charger decreases due to "multiplexing gains". For instance, consider $\lambda=0.5, B=112$ $\mathrm{kWh}$, and two cases for the station size, $N_{1}=50$ and $N_{2}=150$. Further note that power drawn from the grid for $N_{2}=150$ is set to the three times of that $N_{1}=50$ that is $C_{1}=\frac{1}{3} \times C_{2}$. Moreover, outage probabilities are calculated for two cases as $\epsilon_{1}=0.1093$ and $\epsilon_{2}=0.0275$. It is easy to see that gains in outage probability, given by $\epsilon_{1} / \epsilon_{2}=3.97$ is higher than the amount of capacity increase, that is $C_{2} / C_{1}=3$. To that end, storage units lower peak consumption and save electricity bills. 


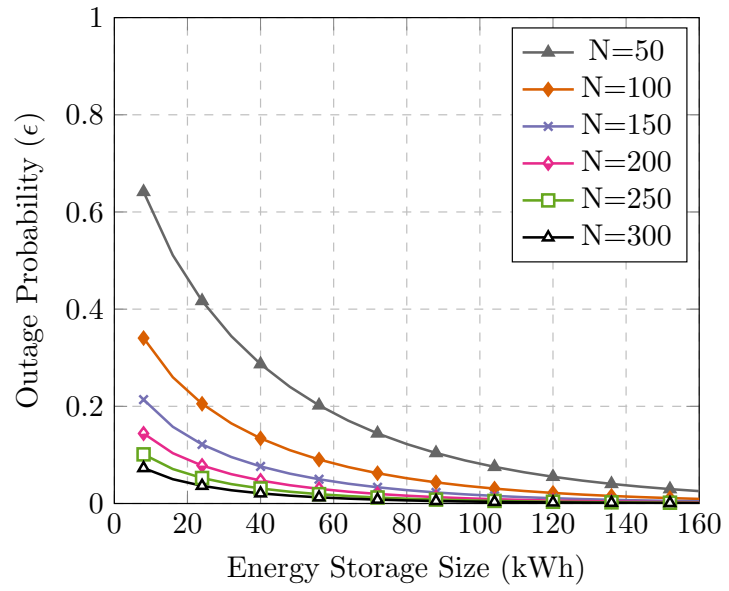

(a) $\lambda=0.25 \mathrm{~N}$

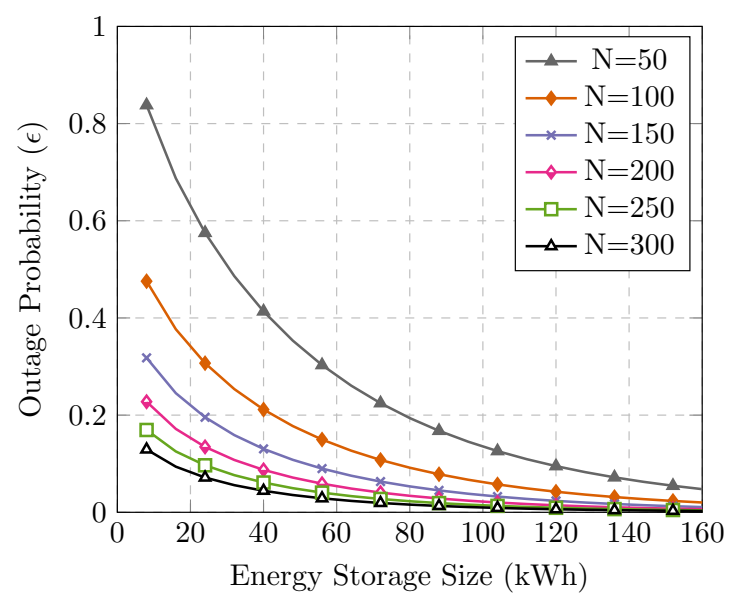

(b) $\lambda=0.50 \mathrm{~N}$

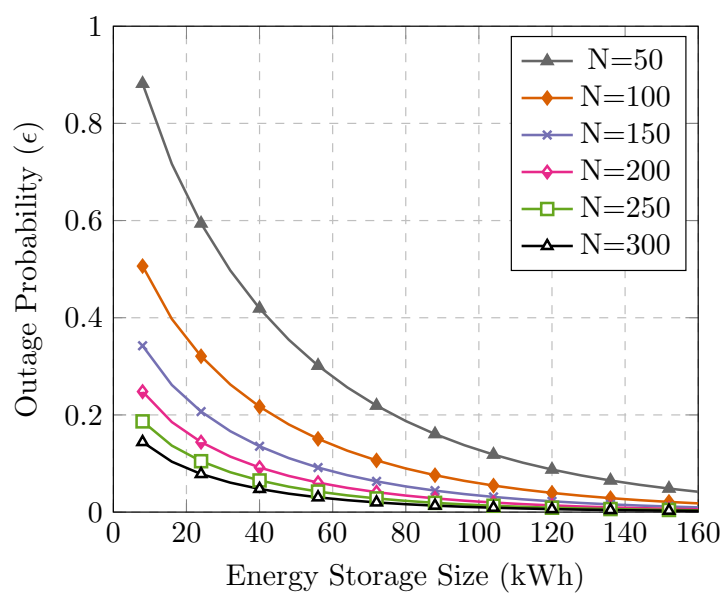

(c) $\lambda=0.75 \mathrm{~N}$

Fig. 5: Energy Storage Size versus outage probability for a varying number of PEV chargers 




(a) $N=100$ chargers.

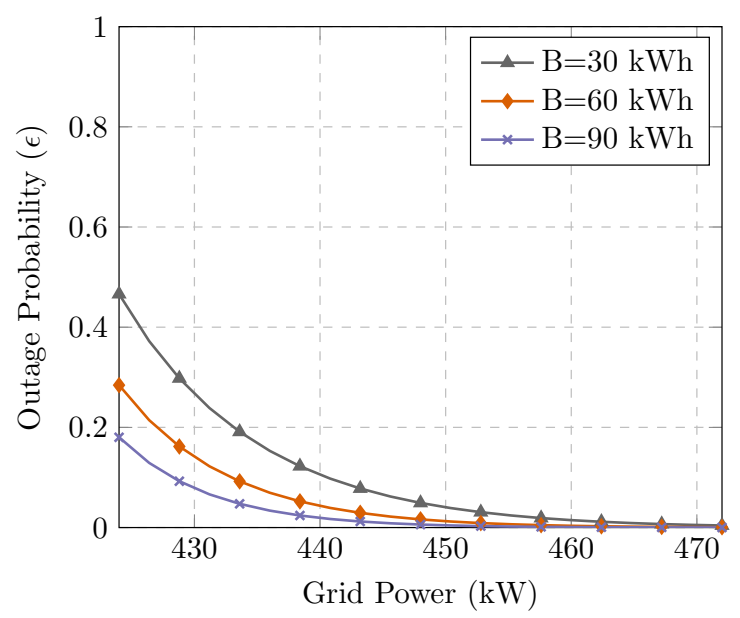

(b) $N=200$ chargers.

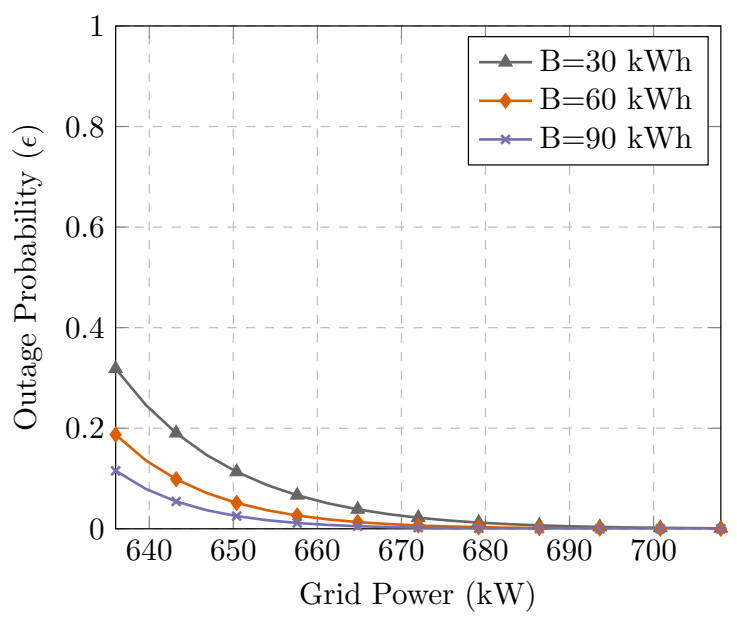

(c) $N=300$ chargers.

Fig. 6: Energy Storage Size versus varying grid power. 


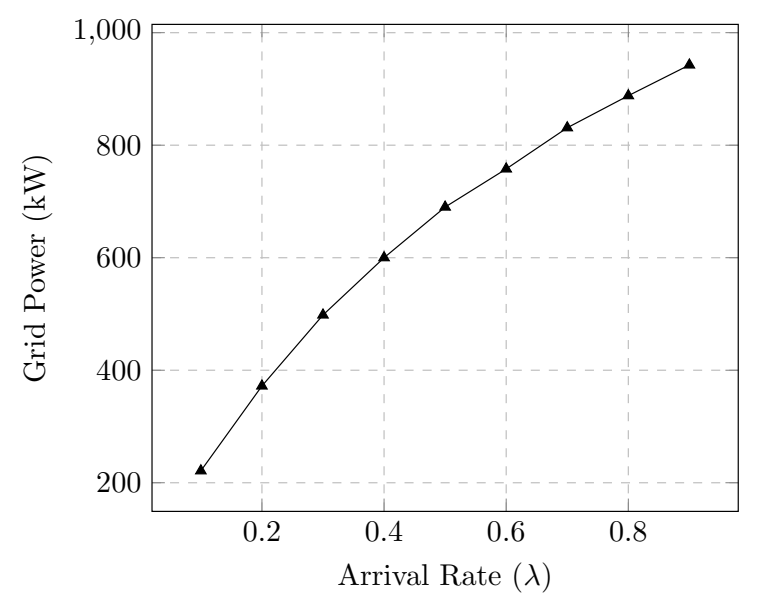

Fig. 7: Minimum amount of power required to provide $\epsilon=0.005$ outage probability with $N=300$ and $B=30 \mathrm{kWh}$.

Next, we evaluate a different case in which we assume that storage size and the number of chargers is known $(N)$ and we are interested in calculating the amount of power to be drawn from the grid to support a target outage probability. In Figure 6, we present results for $\lambda=0.5$ and three different station sizes varying from $N=100$ to $N=300$. From the presented results, system operators can choose appropriate amounts of power for the station. Similar to previous discussion, as the number of chargers increases, the resource requirement per charger decreases. For instance, to provide $\epsilon=0.05$ service for $N=100$ and $B=30 \mathrm{kWh}$, there is a need for a $4.69 \mathrm{~kW}$ per charger. However, for $N=300$, this value reduces to $2.3 \mathrm{~kW}$. As a third evaluation, we calculate the minimum amount of power required to provide $\epsilon=0.005$ outage probability for a station with 300 chargers and $B=30 \mathrm{kWh}$ energy storage (results depicted in Figure 7). It is noteworthy that, in the absence of storage unit, when the arrival rates gets close to $\mu$, i.e., $\lambda \rightarrow \mu$, the station would need to draw $300 \times 6=1800 \mathrm{~kW}$ power. Such findings are the main motivation for the the next case study in which we quantify the economic savings introduced by the energy storage systems.

\section{Charging Station Economic Analyses}

The primary motivation to employ storage units at charging lots is to reduce operational cost and avoid major grid reinforcements. The break down of the monthly electricity bill of a typical 




Fig. 8: Hourly PEV demand for $N=200$ chargers.

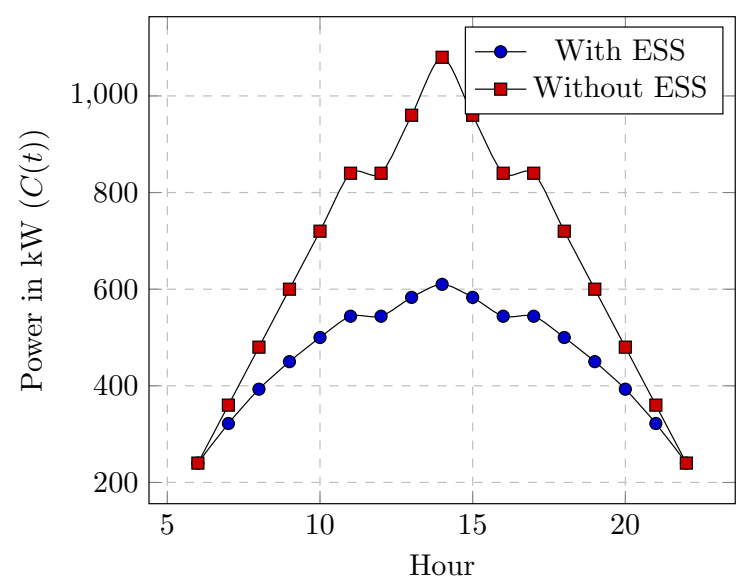

Fig. 9: Hourly power drawn from the grid for 99.5\% reliability.

charging station is composed of three elements: (i) a fixed fee; (ii) energy charges (USD/kWh); and (iii) demand charges (USD/kW) [44]. For this case study, we use actual fees from a utility company in San Diego, CA and three parameters are set as follows. Monthly fixed fee is \$140, energy and demand charges are presented in Tables II and III. Note that demand charge rates are calculated based on the highest level of power the station draws during 15 minutes within each billing month.

We consider that charging station with $N=200$ slots, each equipped with level 2 chargers 
similar as before. The station operates from 6 am to $10 \mathrm{pm}$, that is denoted by $t=1,2, \ldots, 16$. At each hour customer arrival rate varies with time and shown in Figure 8. Energy storage size is chosen according to peak hour and station power $C(t)$ is chosen to provide $\epsilon=0.005$ outage performance for the given energy storage size. Moreover, assume that due to the limitation of the supporting power network station can draw up to $610 \mathrm{~kW}$. To that end, choosing storage unit as $B=108 \mathrm{kWh}$ would suffice outage probability requirements at all times. In addition, the minimum amount of power drawn from the grid for each hour is calculated and presented in Figure 9. In the same figure, we further show the amount of power required to provide similar service in the absence of an energy storage system. It can be seen that there are significant difference in the peak electricity drawn from the grid. To further clarify monetary savings, we calculate monthly electricity bills of a typical charging station when it is subject to an actual time of use electric vehicle plan adopted commercial customer electricity tariffs from Pacific Gas and Electric Company located in California [45] and rates presented in Tables II and III for summer and winter periods.

TABLE II: Commercial Customer Summer (June-September) Energy and Demand Tariffs (USD per kW) adopted from Pacific Gas and Electric [45].

\begin{tabular}{lccc}
\hline & Time Period & Energy Rate & Demand Rate \\
\hline Peak & $4 \mathrm{pm}-9 \mathrm{pm}$ & 0.165 & 25.79 \\
\hline Partial-Peak & $\begin{array}{c}2 \mathrm{pm}-4 \mathrm{pm} \\
9 \mathrm{pm}-11 \mathrm{pm}\end{array}$ & 0.135 & 5.30 \\
\hline Off-Peak & All other hours & 0.114 & N/A \\
\hline
\end{tabular}

TABLE III: Commercial Customer Winter (October-May) Energy and Demand Tariffs (USD per $\mathrm{kW}$ ) adopted from Pacific Gas and Electric [45].

\begin{tabular}{lccc}
\hline & Time Period & Energy Rate & Demand Rate \\
\hline Peak & $4 \mathrm{pm}-9 \mathrm{pm}$ & 0.165 & 21.44 \\
\hline Super Off-Peak & 9 am $-2 \mathrm{pm}$ & 0.07 & N/A \\
\hline Off-Peak & All other hours & 0.114 & 1.77 \\
\hline
\end{tabular}

To that end, monthly electricity bill of the proposed station model is compared with the one that does not employ energy storage system for summer and winter tariffs. In addition to 


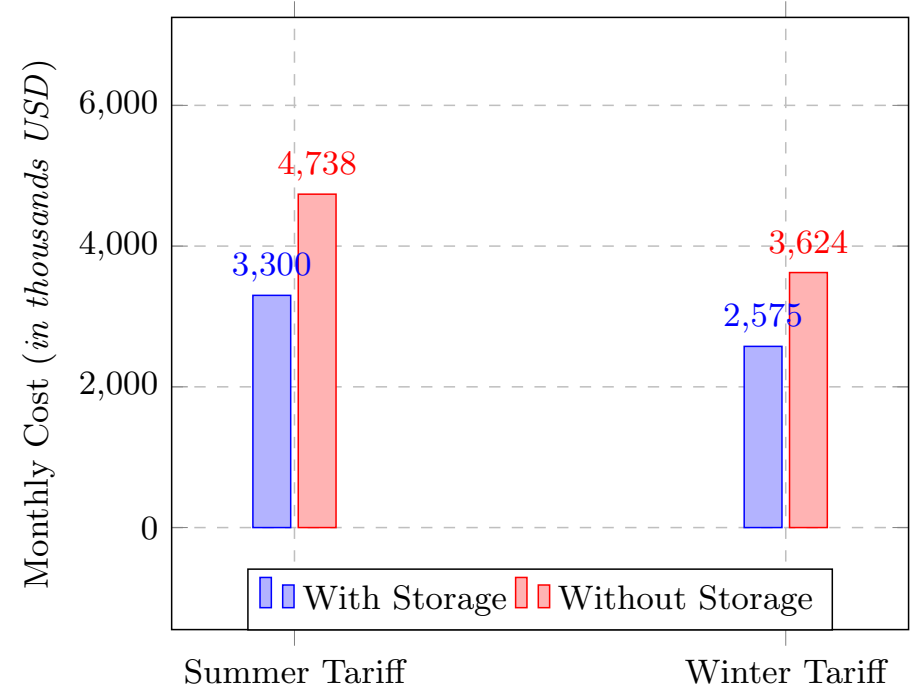

Fig. 10: Comparison of monthly electricity bills based on fix cost, energy cost, and demand cost.

electricity cost, unit energy storage cost (USD/kWh) is included for the "with storage" case. In [46], unit storage cost for a 20 year project is calculated as $169.5 \mathrm{USD} / \mathrm{kWh}$. Moreover, monthly maintenance cost is calculated as $1300 \mathrm{USD}^{2}$. Considering the storage size of $108 \mathrm{kWh}$, normalised cost of storage per month becomes 59.7 USD. Finally, for the given assumptions, charging stations with and without storage units are compared and depicted in Figure 10. It can be seen that employing energy storage system reduces monthly electricity bill by nearly $31 \%$ in summer and $29 \%$ in winter.

It is noteworthy that actual cost-benefit analyses is usually project-specific. As explained above, the monetary calculations depend on customer demand, electricity tariffs, and possible other use case scenarios for the storage unit such as black-out support, reliability improvement, and selling excess energy in the energy market. However, preceding calculations show that on-site storage units are effective in reducing monthly running cost of charging stations.

\section{CONCLUSION}

In this paper, we presented a probabilistic capacity planning methodology for plug-in electric vehicle charging lots equipped with on-site energy storage systems. We modelled the system

\footnotetext{
${ }^{2}$ In [46], the unit storage cost is $150 €$ per kWh. Last three year's average Euro to US Dollar exchange rate is used [47].
} 
with a Markov-modulated Poisson process. System states are represented by (i) number of vehicles in the charging lot and (ii) energy storage charge level. We used a matrix-geometric solution technique to calculate steady-state distributions. The primary motivation was to show the relationship between energy storage size, grid power, and customer statistics. In the last part, we presented a number of case studies to calculate energy storage size and system capacity requirements to provide PEVs a reasonable level of quality of service. The results showed that storage units can be instrumental in reducing peak electricity consumption and associated demand charges. Moreover, as the number of chargers increase the energy storage requirement per charger decreases due to statistical gains. As a future work, we will consider the case where storage unit can participate in energy markets and develop market bidding strategies. 


\section{REFERENCES}

[1] J. Earl, M. J. Fell, Electric vehicle manufacturers' perceptions of the market potential for demand-side flexibility using electric vehicles in the united kingdom, Energy policy 129 (2019) 646-652.

[2] K. MacLellan, G. Faulconbridge, Electric cars win? britain to ban new petrol and diesel cars from 2040 (2017).

[3] I. S. Bayram, A. Tajer, Plug-in electric vehicle grid integration, Artech House, 2017.

[4] C. d. M. Affonso, M. Kezunovic, Probabilisitic sizing of pv generation on commercial parking lot with pevs to avoid transformer aging, in: 2019 IEEE Milan PowerTech, 2019, pp. 1-6.

[5] K. Clement-Nyns, E. Haesen, J. Driesen, The impact of charging plug-in hybrid electric vehicles on a residential distribution grid, IEEE Transactions on power systems 25 (1) (2009) 371-380.

[6] Power networks navigate electric car challenge.

URL https://www.ft.com/content/854526c8-30eb-11e7-9555-23ef563ecf9a

[7] A. Haslett, Smarter charging - a uk transition to low carbon vehicles: Summary report, Tech. rep., Energy Technologies Institute (2019).

[8] Transport scotland: Over 1000 electric vehicle charge points in scotland.

URL https://www.transport.gov.scot/news/over-1000-electric-vehicle-charge-points-in-scotland/

[9] West coast electric highway.

URL http://www.westcoastgreenhighway.com/electrichighway.htm

[10] Global ev outlook," international energy agency, 2019.

URL https://www.iea.org/reports/global-ev-outlook-2019

[11] Electric vehicle outlook 2019.

URL https://about.bnef.com/electric-vehicle-outlook/\#toc-download

[12] M. Smith, J. Castellano, Costs associated with non-residential electric vehicle supply equipment: Factors to consider in the implementation of electric vehicle charging stations, Tech. rep. (2015).

[13] M. C. Falvo, D. Sbordone, I. S. Bayram, M. Devetsikiotis, Ev charging stations and modes: International standards, in: 2014 International Symposium on Power Electronics, Electrical Drives, Automation and Motion, IEEE, 2014, pp. 1134-1139.

[14] X. Liang, S. Srdic, J. Won, E. Aponte, K. Booth, S. Lukic, A 12.47 kv medium voltage input $350 \mathrm{kw}$ ev fast charger using 10 kv sic mosfet, in: 2019 IEEE Applied Power Electronics Conference and Exposition (APEC), 2019, pp. 581-587.

[15] S. S. Williamson, A. K. Rathore, F. Musavi, Industrial electronics for electric transportation: Current state-of-the-art and future challenges, IEEE Transactions on Industrial Electronics 62 (5) (2015) 3021-3032.

[16] M. Yilmaz, P. T. Krein, Review of battery charger topologies, charging power levels, and infrastructure for plug-in electric and hybrid vehicles, IEEE transactions on Power Electronics 28 (5) (2012) 2151-2169.

[17] A. Khaligh, S. Dusmez, Comprehensive topological analysis of conductive and inductive charging solutions for plug-in electric vehicles, IEEE Transactions on Vehicular Technology 61 (8) (2012) 3475-3489.

[18] R. Wang, P. Wang, G. Xiao, Two-stage mechanism for massive electric vehicle charging involving renewable energy, IEEE Transactions on Vehicular Technology 65 (6) (2016) 4159-4171.

[19] K. Seddig, P. Jochem, W. Fichtner, Two-stage stochastic optimization for cost-minimal charging of electric vehicles at public charging stations with photovoltaics, Applied energy 242 (2019) 769-781.

[20] D. Ronanki, S. S. Williamson, Modular multilevel converters for transportation electrification: challenges and opportunities, IEEE Transactions on Transportation Electrification 4 (2) (2018) 399-407.

[21] M. R. Khalid, M. S. Alam, A. Sarwar, M. J. Asghar, A comprehensive review on electric vehicles charging infrastructures and their impacts on power-quality of the utility grid, eTransportation 1 (2019) 100006. 
[22] I. Rahman, P. M. Vasant, B. S. M. Singh, M. Abdullah-Al-Wadud, N. Adnan, Review of recent trends in optimization techniques for plug-in hybrid, and electric vehicle charging infrastructures, Renewable and Sustainable Energy Reviews 58 (2016) 1039-1047.

[23] Y. Liu, Y. Zhu, Y. Cui, Challenges and opportunities towards fast-charging battery materials, Nature Energy 4 (7) (2019) $540-550$.

[24] S. Srdic, S. Lukic, Toward extreme fast charging: Challenges and opportunities in directly connecting to medium-voltage line, IEEE Electrification Magazine 7 (1) (2019) 22-31.

[25] S. Negarestani, M. Fotuhi-Firuzabad, M. Rastegar, A. Rajabi-Ghahnavieh, Optimal sizing of storage system in a fast charging station for plug-in hybrid electric vehicles, IEEE transactions on transportation electrification 2 (4) (2016) 443453.

[26] I. S. Bayram, G. Michailidis, M. Devetsikiotis, F. Granelli, Electric power allocation in a network of fast charging stations, IEEE Journal on Selected Areas in Communications 31 (7) (2013) 1235-1246.

[27] M. R. Sarker, H. Pandžić, K. Sun, M. A. Ortega-Vazquez, Optimal operation of aggregated electric vehicle charging stations coupled with energy storage, IET Generation, Transmission Distribution 12 (5) (2018) 1127-1136. doi:10.1049/ietgtd.2017.0134.

[28] E. Ucer, I. Koyuncu, M. C. Kisacikoglu, M. Yavuz, A. Meintz, C. Rames, Modeling and analysis of a fast charging station and evaluation of service quality for electric vehicles, IEEE Transactions on Transportation Electrification 5 (1) (2019) 215-225.

[29] P. Fan, B. Sainbayar, S. Ren, Operation analysis of fast charging stations with energy demand control of electric vehicles, IEEE Transactions on Smart Grid 6 (4) (2015) 1819-1826.

[30] Z. Fotouhi, M. R. Hashemi, H. Narimani, I. S. Bayram, A general model for ev drivers' charging behavior, IEEE Transactions on Vehicular Technology 68 (8) (2019) 7368-7382.

[31] H. Liang, A. K. Tamang, W. Zhuang, X. S. Shen, Stochastic information management in smart grid, IEEE Communications Surveys \& Tutorials 16 (3) (2014) 1746-1770.

[32] The 50 states of electric vehicles, Tech. rep., NC Clean Energy Technology Center (2018).

[33] G. Fitzgerald, C. Nelder, Evgo fleet and tariff analysis (2017).

[34] P. Fan, B. Sainbayar, S. Ren, Operation analysis of fast charging stations with energy demand control of electric vehicles, IEEE Transactions on Smart Grid 6 (4) (2015) 1819-1826.

[35] T. Ma, O. A. Mohammed, Optimal charging of plug-in electric vehicles for a car-park infrastructure, IEEE Transactions on Industry Applications 50 (4) (2014) 2323-2330.

[36] F. Fazelpour, M. Vafaeipour, O. Rahbari, M. A. Rosen, Intelligent optimization to integrate a plug-in hybrid electric vehicle smart parking lot with renewable energy resources and enhance grid characteristics, Energy Conversion and Management 77 (2014) 250-261.

[37] M. Honarmand, A. Zakariazadeh, S. Jadid, Optimal scheduling of electric vehicles in an intelligent parking lot considering vehicle-to-grid concept and battery condition, Energy 65 (2014) 572-579.

[38] J. Mies, J. Helmus, R. van den Hoed, Estimating the charging profile of individual charge sessions of electric vehicles in the netherlands, World Electric Vehicle Journal 9 (2) (2018) 17.

[39] I. S. Bayram, A. Tajer, M. Abdallah, K. Qaraqe, Capacity planning frameworks for electric vehicle charging stations with multiclass customers, IEEE Transactions on Smart Grid 6 (4) (2015) 1934-1943.

[40] M. F. Neuts, Matrix-geometric solutions in stochastic models: an algorithmic approach, Courier Corporation, 1994.

[41] G. Latouche, V. Ramaswami, Introduction to matrix analytic methods in stochastic modeling, Vol. 5, Siam, 1999. 
[42] B. Hajek, Birth-and-death processes on the integers with phases and general boundaries, Journal of Applied Probability 19 (3) (1982) 488-499.

[43] L. Kleinrock, Queueing systems, volume 1:Theory, Vol. 66, wiley New York, 1975.

[44] I. S. Bayram, M. Ismail, A stochastic model for fast charging stations with energy storage systems, in: 2019 IEEE Transportation Electrification Conference and Expo (ITEC), IEEE, 2019, pp. 1-5.

[45] Pacific gas and electric company.

URL https://www.pge.com/tariffs/index.page

[46] J. Domínguez-Navarro, R. Dufo-López, J. Yusta-Loyo, J. Artal-Sevil, J. Bernal-Agustín, Design of an electric vehicle fast-charging station with integration of renewable energy and storage systems, International Journal of Electrical Power \& Energy Systems 105 (2019) 46-58.

[47] OECD exchange rates.

URL https://data.oecd.org/conversion/exchange-rates.htm 